\title{
Jean-Marc Regnault, Taui ou le pouvoir confisqué
}

Les Éditions de Tahiti, Moorea, 2004

Michel Panoff

\section{CpenEdition}

Journals

Édition électronique

URL : http://journals.openedition.org/jso/494

DOI : $10.4000 /$ jso.494

ISSN : 1760-7256

Éditeur

Société des océanistes

Édition imprimée

Date de publication : 1 décembre 2005

Pagination : 199-201

ISSN : 0300-953x

Référence électronique

Michel Panoff, «Jean-Marc Regnault, Taui ou le pouvoir confisqué », Journal de la Société des Océanistes [En ligne], 120-121 | Année 2005, mis en ligne le 01 septembre 2008, consulté le 24 septembre 2020. URL : http://journals.openedition.org/jso/494 ; DOI : https://doi.org/10.4000/jso.494

(c) Tous droits réservés 


\section{COMPTES RENDUS}

Jean-Marc Regnault, 2004. Taui ou le pouvoir confisqué, Moorea, Les Éditions de Tahiti, 183 p.

Ce petit livre est de la plus grande utilité. Puisque le "système Flosse" est déjà largement connu des océanistes, c'est plutôt comme révélateur de la politique gouvernementale française qu'il sera utile au lecteur métropolitain. En effet, il offre aux Français, dans un miroir grossissant, leur portrait et l'image de leur démocratie telle qu'elle se pratique dans le monde réel (règles et circonscriptions électorales aménagées pour les besoins de la cause, casuistique procédurière déjouant l'inégibilité, etc.). Foin d'exotisme ! C'est bien de nous qu'il s'agit, aux antipodes, dans cette crise largement médiatisée. À l'heure où l'opinion publique se familiarise avec les mystères de la "Françafrique", il est utile de lui montrer que les mêmes attitudes et les mêmes habitudes se retrouvent aussi dans ce minuscule territoire. Comme si, partout, les diverses arrière-cours de la maison "France" étaient invariablement gérées d'une façon identique, que le gérant se nomme Mitterrand ou Chirac.

De manière pédagogique le livre est divisé en trois parties :

$1^{\circ}$ Se familiariser avec la vie politique en Polynésie. L'auteur y rappelle, s'il en était besoin, que généralement la solidarité de parentèle (fetii) l'emporte sur le loyalisme politique, ce qui explique comment Flosse a pu "débaucher" certains adversaires au moment opportun.

$2^{\circ}$ Deux hommes, deux partis, deux Polynésie. C'est la plus fouillée et la plus utile au plan politique. C'est d'elle principalement qu'il s'agira dans ce compte rendu.

$3^{\circ}$ Pratiques coloniales en faveur d'un clan. Cette partie est la plus vivante car elle culmine en une chronique des quatre mois de Taui qui virent se succéder les interventions ministérielles de Brigitte Girardin et les expéditions de secours du Parti socialiste. À sa lecture, on éprouve un fort sentiment de proximité.

C'est en vis-à-vis que l'auteur veut nous peindre les portraits des deux adversaires et le déroulement de leurs carrières, mais c'est par Flosse qu'on le sent particulièrement inspiré et peut-être même fasciné. On peut le comprendre. La mégalomanie, les contrevérités efficaces, la pugnacité et le clientélisme s'étalant au grand jour, tout cela vous a un relief et un éclat que ne peuvent égaler l'honnêteté, la ténacité et l'absence d'esbroufe de Temaru. Une fois celui-ci au pouvoir, c'est la maladresse ou l'incompétence que l'on pouvait reprocher éventuellement à son équipe, ce qui est derechef bien terne, alors que Flosse dans la même position était accusé de détournement de fonds, de prise illégale d'intérêts et de corruption, ce qui ressemble à autant de prouesses sportives. On est impressionné. On s'attacherait presque à ce personnage qui a quelque chose du champion olympique, même quand les préférences idéologiques vous porteraient plutôt de l'autre côté, comme c'est peut-être le cas de Regnault. On voit Flosse, entrant au service de Rudy Bambridge, le tombeur de Pouvanaa, et devenant son homme de confiance en politique comme en affaires. On assiste à son ascension sociale en même temps que grandit son influence politique et que s'aiguisent ses appétits. Pour finir, on se convainc que l'affairisme est consubstantiel au personnage et à son destin. Richissime, il lancera une chaîne de télévision à sa dévotion. Irrésistiblement, on pense à un certain Berlusconi contrôlant milliards et médias. Nombreux sont justement les esprits simples qui se demandent comment Flosse a pu bâtir une si grande fortune en si peu de temps. Regnault se fait l'écho de leurs interrogations mais il n'apporte pas de réponse. Le personnage y gagne évidemment une aura supplémentaire et l'atmosphère régnant à Papeete se fait un peu plus lourde dans l'imagination du lecteur.

Flosse eut l'intelligence de concurrencer les indépendantistes sur leur propre terrain, puis de se présenter comme le seul interlocuteur possible pour le gouvernement de Paris, lequel voulait son $\mathrm{CEP}^{1}$ à tout prix Grands travaux, création de milliers d'emplois, multiplication des liaisons inter-insulaires et formation de nouvelles élites : tels furent les effets induits du CEP Coincés dans un tel engrenage, les indépendantistes étaient condamnés à l'impuissance, comme le reconnaissait Teariki par exemple en 1975 (p. 32). Seul un virtuose du double langage comme Flosse pouvait prospérer en se déclarant pour une "autonomie dure" contre une "indépendance molle" (p. 37). La formule était bien trouvée et annonçait la suite logique de l'histoire qui sera faite de surenchères et de chantages à répétition toujours ostensiblement dans le cadre de la République. À tout coup, il était gagnant puisqu'il jouait simultanément sur la corde anti-colonialiste et sur la corde poujadiste d'exécration des représentants de l'État. 
Il obtint donc l'autonomie, qui lui assura un pouvoir absolu sur le pays. Dès lors, la métropole ne pouvait plus se mêler de ses affaires, ce qui signifiait pratiquement impunité devant les tribunaux, absence de contrôle sur l'utilisation de l'argent public, liberté de discriminer les candidats aux emplois et aux logements, etc. En voici une conséquence parmi d'autres : grâce à son long exercice du pouvoir, il a graduellement obtenu le ralliement de quarante-deux maires (sur un total de quarante-huit) qui sont devenus ses "clients" et restent dans sa mouvance, résultat tout à fait décisif pour le quadrillage de la population (p. 111). Là où le RDPT $^{2}$ rêvait de l'indépendance pour introduire un peu de justice et d'égalité en limitant l'exploitation capitaliste, rêve épisodiquement repris par Temaru, Flosse utilise au contraire l'autonomie pour empêcher l'application des 35 heures et de la législation sociale, imposer le blackout sur le chômage et interdire toute innovation fiscale qui s'inspirerait de la métropole. On se souviendra ici que les partisans de l'Algérie française, eux aussi, avaient rêvé un moment de rompre les liens avec la France pour échapper à des lois et à des contrôles qui handicapaient leur libéralisme pur et dur.

Il y a 50 ans, la métaphore qui faisait florès dans les cercles du pouvoir et dans les dîners mondains était celle de la danseuse. La France est assez riche et assez raffinée, disait-on en parlant de Tahiti, pour s'offrir une danseuse. Après l'installation du CEP, c'est d'autres images et d'autres mots, comme proxénétisme ou chantage, qui sembleraient convenir à la situation. À moins que ce ne soit plutôt ce cri du cœur d'un secrétaire d'État aux DOM-TOM : "Croit-on donc que la République est un guichet?» Autre image, même scandale.

Pas d'autonomie sans argent, martelait Flosse. Il obtint donc aussi l'argent, et d'autant plus d'argent qu'il fallait prévoir à long terme les dommages causés par le CEP et les réparer. Dommages matériels, voire sanitaires, s'entend, car des dommages sociétaux et moraux, hormis quelques responsables protestants, personne n'en avait cure face à la raison d'État. Ils allaient se manifester plus tard, notamment sous la forme des émeutes de 1995 ou de cette alternance inattendue de 2004.

On a déversé tant de milliards sur ce petit territoire que tout a changé de nature, et pas seulement de proportions. Rien de plus révélateur que les insultes et les grossièretés proférées par les gens au pouvoir contre leurs adversaires dans les médias et à l'Assemblée de Polynésie car ils vont directement à l'essentiel: le rapport de force, qui rend oiseuse toute discussion. Et si, par malheur, le rapport de force vient à changer, la situation tourne à la farce comme pendant les quatre mois du Taui (changement), quand Flosse manœuvre en accord avec le gouvernement Raffarin pour faire tomber Temaru. Là, on mesure bien tout ce que la classe politique locale a pu gagner en un demi-siècle de coopération nucléaire franco-tahitienne !
Pour imaginer l'esprit qui animait cette coopération, on retiendra l'invention hilarante de Charles Hernu qui décida que l'État paierait des droits de douane sur le matériel militaire du CEP entrant en Polynésie. Il s'agissait d'offrir 2 milliards de plus à Flosse alors que la France était au ban des nations avec le traité de Rarotonga et l'affaire du Rainbow Warrior (pp. 94-95). Il était tellement plus facile de traiter avec lui qu'avec les socialistes polynésiens, camarades de parti se réclamant aigrement de l'anticolonialisme quand l'heure était au patriotisme ! Dommage que les paysans du Larzac luttant contre les empiètements de l'Armée n'aient pas bénéficié de pareils expédients douaniers pour se remplumer et pour valoriser leur élevage extensif!

Parmi les révélations de Regnault, celles qui concernent la mégalomanie de Flosse et les moyens de la satisfaire sont particulièrement éloquentes. Ainsi, à propos du fonctionnement du "château", ce sont 629 fonctionnaires, pas moins, qui sont attachés au président Flosse (p. 172). Rapporté au nombre d'habitants, ce chiffre fait paraître presque décent, voire modeste, celui des 1700 personnes qui s'affairent à l'Elysée au service du président Chirac. De même, on pourra admirer la réalisation pharaonique de la mairie de Pirae dont le projet fut évoqué dès 1966 dans un donnant-donnant explicite lors des premiers tirs atomiques (p. 80).

Avec la vivacité du journaliste qui interviewe les politiciens, Regnault combine la rigueur de l'historien universitaire qui déniche des documents probants dans les archives, et il rend fort crédible son explication de la "crise polynésienne". Pour lui, elle découle de la volonté de maintenir coûte que coûte au pouvoir l'homme qui pendant vingt-cinq ans a été le partenaire des dirigeants et des hommes d'affaires français en même temps que le bienfaiteur d'une immense "clientèle". De surcroît, membre du gouvernement Chirac de 1986 à 1988, il a été de toutes les opérations, il connaît tous les dossiers sensibles. Il avait acquis le droit imprescriptible de gouverner. Si les urnes donnaient la victoire à son adversaire, les urnes avaient tort.

Taui est le nom emblématique de Temaru et de ses partisans, c'est aussi le nom du changement qu'attendent de nombreux Polynésiens. Ils ne sont pas près de l'obtenir. En tout cas, ce livre ne semble pas le prévoir pour bientôt. Si bien que Regnault a certes rempli sa mission quand il nous montre le "pouvoir confisqué", sous-titre de son ouvrage, mais il nous laisse sur notre faim quant à la possibilité de changement réel dans la société. On aurait pourtant cru qu'elle était visée dans son titre principal. Or, l'affrontement de Flosse et Temaru pour la présidence apparaît finalement comme un épiphénomène qui doit être remis à sa juste place. Ce que l'auteur ne fait pas toujours. Emporté par son récit, il perd parfois le sens des proportions et il lui arrive de mettre la crise actuelle sur le même plan que l'action révolutionnaire de Pouvanaa et son écra-

2. NDLR. Le Rassemblement démocratique des populations tahitiennes, créé en 1949 par Pouvana a Oopa, leader nationaliste. 
sement en 1958, ou que l'arrivée de la LMS $^{3}$ en 1797 (p. 176).

En conséquence, la description de la crise fait la part belle aux stratégies politiciennes et à la psychologie des deux protagonistes, toutes choses que Regnault analyse avec finesse, mais qui régaleront surtout l'accro des médias qui "affriole" anecdotes pittoresques ou one-man show dramatique. Au bout du compte, c'est forcément au sociologue et au militant que doit revenir le sujet extraordinairement difficile du changement en profondeur, et non au spécialiste de science politique. Attendons de voir ce qu'ils en feront après quarante années galopantes de modernisation exogène !

L'auteur dédie son travail aux humbles, aux petits, à ceux que l'on n'entend jamais (p. 3). Il voudrait qu'ils puissent avoir enfin la parole. Qui ne s'associerait à son vœu? Et, de fait, l'image sempiternelle de Tahiti comme Paradis, comme île bienheureuse où n'existeraient ni conflits ni désespoir et qui fait rêver les touristes, est une invention des hommes d'influence, véhiculée par les hommes d'influence. On n'a jamais demandé aux Tahitiens les plus humbles s'ils s'y reconnaissaient. D'ailleurs, même Oscar Temaru ne voit probablement pas les choses comme eux. Malgré les valeurs qu'il incarne et le courage qui l'anime, il fait partie des privilégiés avec des revenus mensuels en 2001 dépassant 200000 de nos francs (p. 76).

$\mathrm{Si}$ bien que le duel Flosse/Temaru et cette crise spectaculaire qui ont passionné les médias risquent d'apparaître un jour prochain comme un théâtre d'ombres sans rapport avec les problèmes dans lesquels se débat la masse de la population, notamment celle qui vit ou survit sur le "côté montagne".

Comme le citoyen de métropole a aussi son mot à dire, à l'égal de $\mathrm{M}^{\mathrm{me}}$ Girardin, il osera considérer la folle prodigalité du bailleur de fonds et le gaspillage cynique du bénéficiaire et il en viendra peut-être à souhaiter la rupture du lien pervers qui unit la France à ce territoire "autonome". Du coup, il rêvera que la victoire de Temaru annonce le commencement de la fin, ou au moins un peu de démocratie et un autre partage des richesses?

Ce livre connaîtra certainement une nouvelle édition $^{4}$, il la mérite. Mais il faudra que l'auteur le remanie en un ensemble plus cohérent. Aujourd'hui, il ressemble à un tifaifai $^{5}$ bigarré, avec des morceaux empruntés à ses publications antérieures et d'autres fraîchement rédigés dans le feu de l'actualité. On comprend qu'il se soit hâté de produire son travail. Mais le calme revenu, nul doute qu'il prendra le temps de coordonner les éléments disparates, de soigner la mise en page, de reformater la bibliographie qui est peu lisible et enfin de créer un index des noms propres nous guidant vers les faits et dits des Vernaudon, Léontieff et autres Didier Julia (le fameux spécialiste chiraquien des problèmes exotiques !)

\section{Michel PANOFF}

Réséda Ponga et Laurence LAGABRIELle, 2005. Mèyènô. Conte kanak en français-a’jië, Nouméa, Éd. Grain de sable jeunesse et Centre culturel Tjibaou, 28 p., illustrations, lexique, $\mathrm{CD}$ audio bilingue français-a'jië.

Cet ouvrage est le deuxième album d'une série de contes kanak contemporains, illustrant l'émergence d'une littérature destinée à la jeunesse calédonienne, initiée par l'Agence de développement de la culture kanak et les éditions Grain de sable jeunesse. En associant un texte bilingue, ajië-français, cet album permet au lecteur de se familiariser avec le patrimoine culturel et linguistique kanak, à travers une création contemporaine ${ }^{6}$. Dans ce cadre, la présence dans cet ouvrage d'un CD également bilingue est une bonne initiative qui permet au jeune lecteur de se familiariser avec l'une ou l'autre des langues. Mais revenons rapidement à l'histoire.

Méyènô est un garçon kanak très curieux, en quête de savoir comme nombre de garçons de son âge, et qui vit avec son grand-père Mörua aux pays des ruisseaux. Méyènô signifie d'ailleurs en ajië celui qui « recherche la parole » alors que Mörua est celui qui a vécu longtemps. Comme tous les enfants, il ne cesse de poser des questions à son grand-père sur le monde qui l'entoure. Mais les réponses de son grand-père ne lui suffisent pas et il décide un jour de partir à la découverte de ce monde et de suivre la rivière jusqu'à la mer... pour savoir où elle va! Et le chemin suivi a tout d'un itinéraire kanak.

En effet, si cette quête peut paraître universelle, elle est également très kanak et les thèmes abordés ne sont pas sans rappeler ceux que l'on trouve dans la tradition orale: de l'eau source de vie, à la mer domaine du pays des morts, en passant par le lézard animal totémique, l'importance des ancêtres et des esprits des morts qui supportent les vivants et leurs actions... En arrivant au bord de mer, Méyènô est attiré par les profondeurs. Le lézard prévient le grandpère qui part pour retrouver son petit-fils au pays des morts. Cette quête est donc aussi un voyage au pays sous-marin des morts ! Et le grand-père et son petit-fils se retrouvent pour l'éternité au pays des morts avec tous leurs ancêtres.

Cette histoire est très joliment accompagnée d'illustrations aux tons chauds, rendant à merveille l'atmosphère des paysages calédoniens avec des personnages en silhouette, sans traits visibles, permettant à tout

3. NDLR. London Missionary Society.

4. NDLR. Ce livre est aujourd'hui épuisé dans son édition tahitienne. Mais il a été réédité en 2005 en métropole sous le titre Le pouvoir confisqué en Polynésie française. L'affrontement TemarulFlosse, Paris, Les Indes savantes, coll. Asie.

5. NDLR. Couverture polynésienne en patchwork.

6. Le premier ouvrage, Téâ Kanaké, était bilingue paicî-français et reprenait un grand classique de la tradition orale paicî. 